BABELAO 8 (2019), p. 1-4

(C) ABELAO (Belgium)

\title{
Une petite tablette néo-sumérienne d'Umma de la collection Van de Sande
}

Par

\section{Camille Lecompte et Axel Van de Sande}

CNRS - UMR 7041, ArScAn-Nanterre ; Louvain-la-Neuve

\begin{abstract}
Pet article propose l'édition d'une tablette appartenant à la collection d'Axel Van de Sande (Louvain-la-Neuve), datant de l'époque de la Troisième Dynastie d'Ur $\left(21^{\mathrm{e}}\right.$ siècle av. J.-C.) et provenant d'Umma. Authentifiée en 1914 par A. Clay, elle fit partie d'une collection américaine, non identifiée ${ }^{1}$.
\end{abstract}

\section{1. Édition de la tablette Van de Sande 1}

Origine : Umma

Date : Šulgi 34/12/-- (vers 2058 av. J.-C. selon la chronologie moyenne)

Contenu : reçu par Lu-girizal de 18000 litres d'orge venant de Iddin-Iškur, champ Kamsalla.

\footnotetext{
${ }^{1}$ Les abréviations se conforment à celles du site CDLI, http://cdli.ox.ac.uk/wiki/abbreviations_for_ assyriology.
} 


\begin{tabular}{|c|c|c|}
\hline Face & Translittération & Traduction \\
\hline 1. & 60 še gur lugal & 18000 litres d'orge au gur royal, \\
\hline 2. & ki $I-d i_{3^{-}}{ }^{\mathrm{d}} / \mathrm{Iškur} \mathrm{ta}$ & de Iddin-Iškur \\
\hline 3. & $\mathrm{Lu}_{2}$-giri ${ }_{17}$-zal & Lu-girizal \\
\hline 4. & šu ba-ti & a reçu \\
\hline \multicolumn{3}{|c|}{ Revers } \\
\hline 1. & a-ša ${ }_{3} \mathrm{Kam}-\mathrm{sal}_{4^{-}}{ }^{\Gamma} \mathrm{la}^{\top}$ & champ (de) Kamsalla \\
\hline 2. & iti ${ }^{\mathrm{d}}$ Dumu-zi & mois 12 \\
\hline 3. & mu An-ša-an $/{ }^{k i}$ ba-bul & année : Anšan fut détruit \\
\hline
\end{tabular}

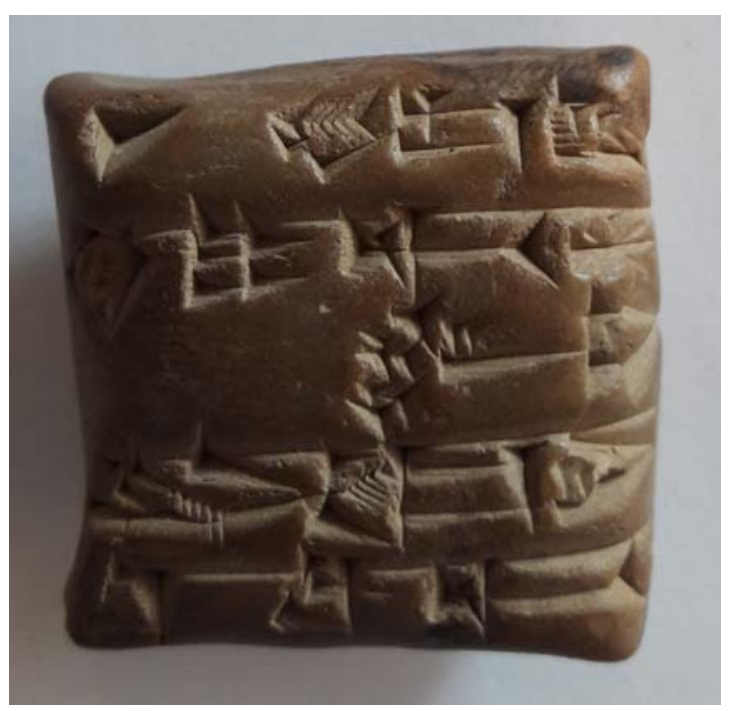

Face, photographie

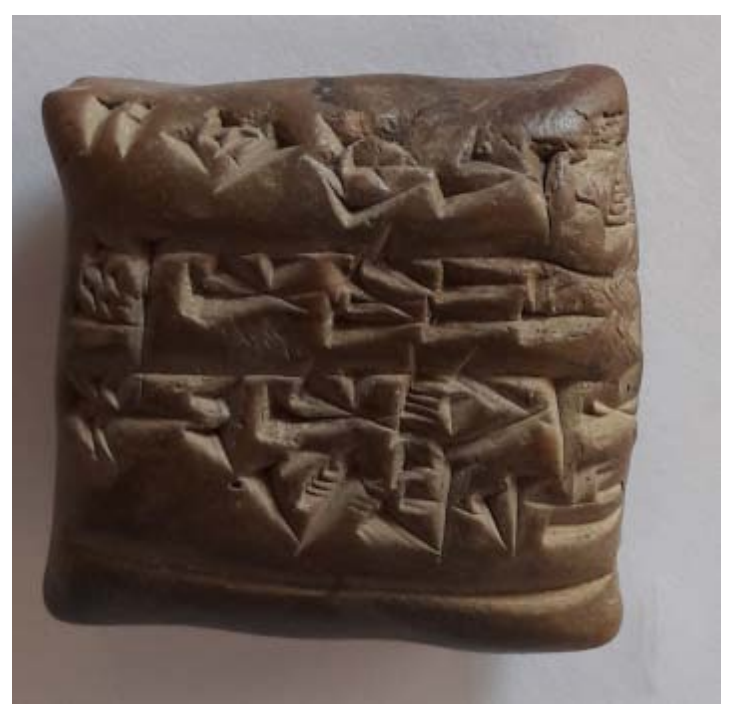

Revers, photographie

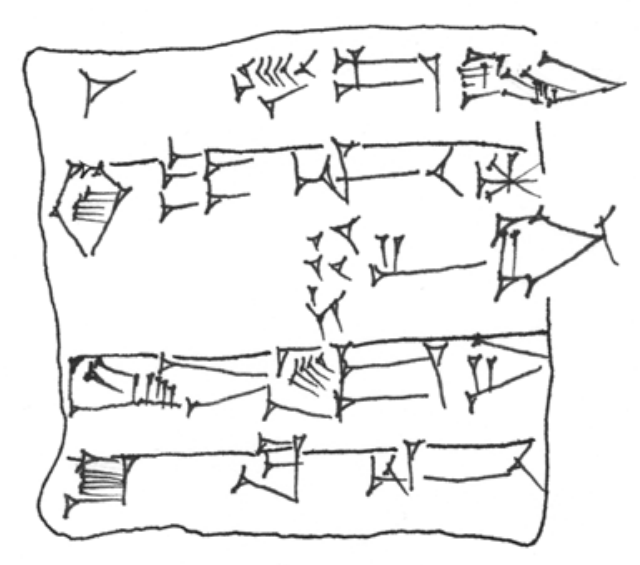

Face, autographie

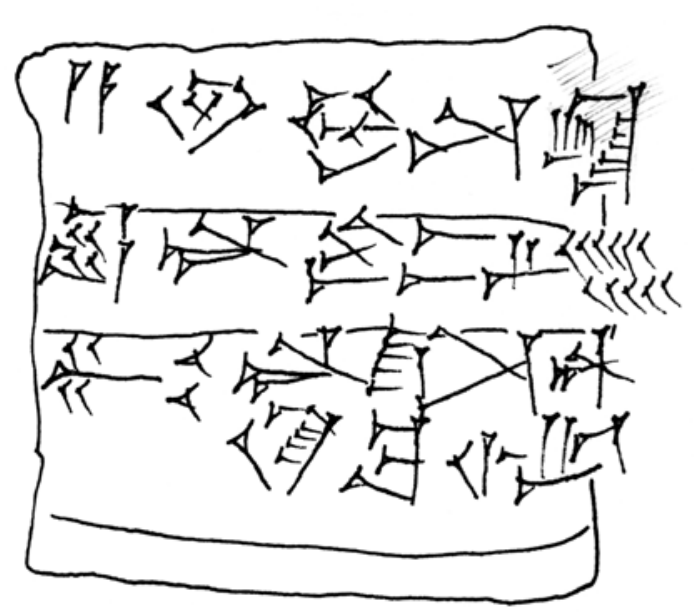

Revers, autographie 


\section{Commentaires}

Face.

2-3. Sur la graphie $I-d i_{3}{ }^{-}{ }^{d}$ Iškur pour Iddin-Iškur, voir, entre autres, HILGERT 2002, p. 57 et 299. Sur le nom sumérien $\mathrm{Lu}_{2}$-giri ${ }_{17}$-zal, fort commun sous Ur III, voir LIMET 1968, p. 235 236 et 478. Ces deux anthroponymes apparaissent certes fréquemment dans les textes d'époque Ur III provenant d'Umma, mais jamais, ou presque, sur un même document, situation qui ne favorise pas l'identification, sur la base d'arguments prosopographiques, d'Iddin-Iškur ${ }^{2}$. Quant à $\mathrm{Lu}_{2}$-giri ${ }_{17}$-Zal, il peut être identifié avec un scribe, connu par son sceau apposé sur de nombreuses tablettes d'Umma, qui gérait des équipes de travailleurs et des productions agraires ${ }^{3}$.

Revers.

1. Le champ a-ša $\mathrm{K}_{3} \mathrm{Kam}-\mathrm{sal}_{4}-\mathrm{la}$, se rapportant à la localité Kamsalla, située dans l'arrièrepays d'Umma ${ }^{4}$, ne semble attesté que dans trois autres documents, MVN 21, 298, face. 3, SAT 2, 42, face 5 , où il est également associé à des quantités de céréales ${ }^{5}$, ainsi que dans RA 62, p. $14 \mathrm{n}^{\circ} 21$ (= DAS 362), qui le mentionne avec des travailleurs. Les sources d'Umma, qui donnent davantage d'informations sur l'implantation de Kamsalla elle-même, témoignent de son activité dans le domaine du transport fluvial ${ }^{6}$, ainsi que de son importance dans la maillage rural, notamment par la présence d'un entrepôt guru 7 ou d'une aire de battage ${ }^{7}$.

${ }^{2} I-d i_{3}{ }^{\mathrm{d}}{ }^{\mathrm{d}}$ škur ne saurait en effet être identifié avec l'un des individus de même nom apparaissant à Umma : seul l'un d'eux, mentionné notamment sous Šulgi, reçoit des quantités de céréales, toutefois nettement inférieures à notre texte : voir SAT 2, 198, face 3 et revers 1 ; AAICAB 1/2, Ashm. 1971-377, revers 2-3 (les deux textes étant datés de Šulgi 38).

${ }^{3}$ L'un des administrateurs mentionnés à Umma et portant le nom de $\mathrm{Lu}_{2}$-giri ${ }_{17}$-zal est en effet le bénéficiaire, notamment sous Šulgi, de quantités élevées de céréales chargées depuis la localité Kamsalla : ASJ 16, 107 - 10 , revers 10 ; BPOA 2, 2007, revers 4 ; SANTAG 7, 158, revers 2 ; voir également USC 6770, revers 15 (travailleurs agricoles chargeant de l'orge sur des bateaux depuis Kamsalla). Nous savons par plusieurs tablettes et empreintes de sceaux que $\mathrm{Lu}_{2}$-giri ${ }_{17}$-Zal, d'ailleurs mentionné à de nombreuses reprises, occupait la fonction de dub-sar, scribe, et était le fils de Da-DU-mu. Voir, à propos du rôle de $\mathrm{Lu}_{2}$-giri ${ }_{17}$-zal dans la gestion de la maind'œuvre agricole, STUDEVENT-HICKMAN 2006, volume 2, p. 388-390.

${ }^{4}$ Voir RGTC 2, p. 90-91 ; SAUREN 1966, p. 217 et STEINKELLER 2007, p. 193.

${ }^{5}$ Ces quantités s'y avèrent inférieures à celle de notre texte.

${ }^{6}$ Cf. STEINKEller 2001, p. 38 note 57, p. 38 note 64, p. 51 note 116 et p. 79.

${ }^{7}$ Voir SAUREN 1966, p. 92, 123, 124, 155 et 168, qui mentionne également un «palais » $\mathrm{e}_{2}$-gal de cette localité. 


\section{BIBLIOGRAPHIE}

HiLgert 2002 : M. HiLgert, Akkadisch in der Ur III-Zeit (IMGULA 5), Münster.

Limet 1968 : H. Limet, L'anthroponymie sumérienne dans les documents de la $3^{e}$ dynastie d'Ur, Liège.

SAuren 1966 : H. SaURen, Topographie der Provinz Umma nach den Urkunden der Zeit der III. Dynastie von Ur. Teil I: Kanäle und Bewässerungsanlagen, thèse de doctorat, Heidelberg.

STEINKELler 2001 : P. STEINKELLER, « New Light on the Hydrology and Topography of Southern Babylonia in the Third Millennium », Zeitschrift für Assyriologie, 91, p. 22-84.

SteInkeller 2007 : P. Steinkeller, «City and Countryside in Third Millennium Southern Babylonia », dans E. C. Stone (éd.), Settlement and Society, Essays Dedicated to Robert McCormick Adams, Los Angeles - Chicago, p. 185-211.

Studevent-Hickman 2006 : B. Studevent-Hickman, The Organization of Manual Labor in Ur III Babylonia, thèse de doctorat, Harvard. 